\title{
The use of self infused intravenous immunoglobulin home therapy in the treatment of acquired chronic demyelinating neuropathies
}

\author{
W A C Sewell, V M Brennan, M Donaghy, H M Chapel
}

\begin{abstract}
High dose intravenous immunoglobulin therapy is becoming the established maintenance treatment for several neurological conditions. Therapeutic immunoglobulin is expensive and its administration is time consuming. Efficacy is variable and must be closely monitored. Here, we show that self infused immunoglobulin at home, combined with daily functional strength diaries to predict dose and dosage intervals, is effective, time saving, and convenient for both patients and medical staff.
\end{abstract}

(F Neurol Neurosurg Psychiatry 1997;63:106-109)

Keywords: chronic demyelinating neuropathy; intravenous immunoglobulin; home therapy

The use of intravenous immunoglobulin (IVIg) to treat neurological disease has expanded over recent years, as case reports and then controlled trials have shown its benefits in various conditions. The treatment of chronic inflammatory demyelinating polyneuropathy, ${ }^{12}$ GuillianBarré syndrome, ${ }^{3}$ Lambert Eaton myasthenic syndrome, ${ }^{4}$ dermatomyositis, ${ }^{5}$ and multifocal motor neuropathy ${ }^{6-10}$ with IVIg has been proved to be effective in placebo controlled, double blind clinical trials. Effectiveness of IVIg is also reported for pure motor demyelinating polyneuropathy. ${ }^{6}$

In most studies of the treatment of neuropa-

Department of

Immunology, John

Radcliffe Hospital, Oxford OX3 9DY, UK

W A C Sewell

V M Brennan

M Donaghy

H M Chapel

Correspondence to: Dr H M Chapel.

Received 16 October 1996 and in revised form 14 February 1997

Accepted 3 March 1997 thies with IVIg, a high dose (immunomodulatory) regimen has been used, rather than the replacement doses of $400 \mathrm{mg} / \mathrm{kg}$ used to treat immunodeficient patients. Typical doses have been $400 \mathrm{mg} / \mathrm{kg}$ of immunoglobulin daily for five days, giving a cumulative dose of $2 \mathrm{~g} / \mathrm{kg}$, repeated at four to 10 week intervals. There are several major difficulties with this form of treatment. Firstly, the need to spend time in the hospital dayroom or ward for the patient, who may find that his or her neurological disability makes daily visits to hospital difficult. Secondly, the schedule needs to be tailored to the patient's disability, as rigid administration schedules may result in loss of motor function before the next infusion. For example, an eight weekly infusion protocol almost invariably results in unacceptable loss of functional strength before the next infusion is due. Thirdly, the time spent in travelling to hospital for IVIg therapy may be substantial, and is obviated by self infusion of immunoglobulin, by the patients, in their own homes.

Although home therapy is usually used for replacement doses of intravenous immunoglobulin, in this paper we discuss the feasibility of high dose IVIg as home therapy or as a day case hospital procedure over 24 to 48 hours, the side effects, and the tailoring of the dosing schedule to the individual patient ensuring maximum benefit. There is extensive experience with using self infused IVIg home therapy in immunodeficient patients, with 360 home infusion patients currently on the United Kingdom Home Therapy Register (VM Brennan, personal communication). A prospective study over two years in the United Kingdom has shown that these programmes, as constituted, are safe. ${ }^{12}$ Such home therapy, however, requires formal training in a recognised centre, and registration with the United Kingdom Home Therapy Register, so that this use of IVIg is monitored.

\section{Case report}

The patient was a 62 year old man with an eight year history of symmetric pure motor demyelinating neuropathy; full details are given in Donaghy et al. ${ }^{6}$ Five years ago he began treatment with high dose intravenous immunoglobulin. This was initially started as an inpatient, using immunoglobulin at $400 \mathrm{mg} / \mathrm{kg}$ daily for five days, at intervals of six to eight weeks. There was relief of symptomsalthough with frequent breakthrough weakness towards the end of the interval between infusions (after four weeks).

He was transferred to outpatient infusions, given at the same cumulative dose of immunoglobulin of $1 \mathrm{~g} / \mathrm{kg}$ for each of two days, but 


\begin{tabular}{|c|c|c|c|c|c|c|c|c|}
\hline \multirow[b]{2}{*}{ Sex } & \multirow[b]{2}{*}{ Diagnosis } & \multirow[b]{2}{*}{$\begin{array}{l}\text { Original dose } \\
(g / y)\end{array}$} & \multirow[b]{2}{*}{$\begin{array}{l}\text { Final dose } \\
(g / y)\end{array}$} & \multirow[b]{2}{*}{$\begin{array}{l}\text { Original infusion } \\
\text { time }(h / y)\end{array}$} & \multirow[b]{2}{*}{$\begin{array}{l}\text { Final infusion } \\
\text { time }(h / y)\end{array}$} & \multirow[b]{2}{*}{$\begin{array}{l}\text { Final infusion schedule } \\
\text { (g/weeks) }\end{array}$} & \multicolumn{2}{|c|}{$\begin{array}{l}\text { Days in } 3 \text { month period at maximum } \\
\text { strength (from daily diaries) }\end{array}$} \\
\hline & & & & & & & $\begin{array}{l}\text { Original schedule } \\
(\%)^{\star}\end{array}$ & $\begin{array}{l}\text { Final schedule } \\
(\%) t\end{array}$ \\
\hline$M$ & MMN & 768 & 816 & 768 & 119 & $48 / 3$ & 45 & 0 \\
\hline$M$ & $M M N$ & 720 & 1275 & 192 & 170 & $72 / 3$ & 30 & 0 \\
\hline $\mathrm{F}$ & MMN & 504 & 936 & 336 & 130 & $72 / 4$ & 30 & 4 \\
\hline $\mathrm{F}$ & PMDN & 1248 & 1728 & 364 & 252 & $48 / 2$ & 51 & 18 \\
\hline $\mathrm{F}$ & MMSDN & 480 & 442 & 30 & 48 & $36 / 4$ & 26 & 0 \\
\hline
\end{tabular}

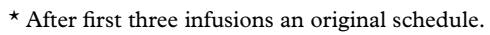

† Last three months; now stable.

$\mathrm{MMN}=$ multifocal motor neuropathy; $\mathrm{PMDN}=$ pure motor demyelinating neuropathy; MMSDN=mixed motor and sensory demyelinating neuropathy; $\mathrm{SAN}=$ sensory ataxic neuropathy.

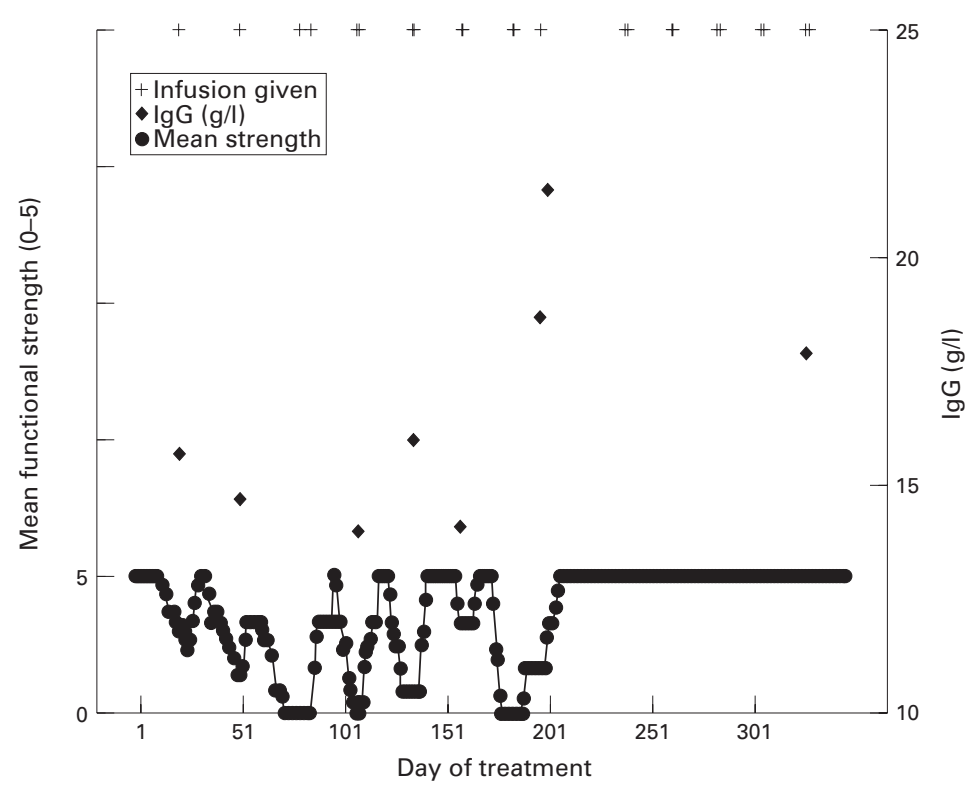

Typical mean functional strength (where maximum function scores 5, and minimum scores zero; arithmetic mean of several activities plotted) against serum IgG concentration for one patient. Immunoglobulin infusion days are marked. There is no apparent correlation

between functional strength and serum IgG concentration. As the spacing of infusions was adjusted to cope with fluctuations in functional strength, control improved until it reached a steady state. ness to treat debilitating weakness-for example, an extra infusion was given five days after an episode of influenza.

\section{Experience with four other patients}

Four other patients (aged 18-50 years) were selected for self infusion at home according to criteria already published. ${ }^{11}$ The table shows the number of hours per year taken to infuse immunoglobulin at home compared with hospital for these four patients. In all cases the time spent infusing was greatly reduced by infusing at home, and there was substantial additional time saved by avoiding the need to travel to the hospital for infusions.

The increased amount of immunoglobulin given per year has maintained the overall functional ability of patients, by raising the serum IgG concentration (figure). The reduced interval of infusions gives each patient a much smoother clinical course (figure), which is presumed to be related to the increased stability of this IgG concentration over time. As the half life of IgG in the serum is three to four weeks, longer intervals between infusions allow the serum IgG concentration to drop below an "efficacious level"; the rate of catabolism of IgG and the concentration of IgG at which efficacy is maintained vary with each patient.

with a reduced interval between infusions (three weeks); this resulted in an increase of dose to $2.50 \mathrm{~g} / \mathrm{kg} / \mathrm{month}$. He was trained in self administration of immunoglobulin in the outpatient clinic. During an 18 week period, during which he received six infusions with the specialist immunology nurse, he and his wife were trained in all aspects of the administration of intravenous immunoglobulin, including aseptic reconstitution of the immunoglobulin product, venepuncture, and taking blood samples through the butterfly needle. The side effects and safety profile of IVIg, and recognition of and action to take in the event of an adverse reaction were fully discussed.

He now administers his own immunoglobulin (48 g) in a single day, monitoring his strength daily using a record of an individually tailored set of tasks (discussed below). These are recorded in a functional strength diary which is reviewed regularly by the immunology and neurology teams. Regular review and dose adjustment if appropriate has maintained stable functional abilities at a maximum. It has been necessary to increase the dose temporarily during periods of unrelated, intercurrent ill-

\section{Current treatment protocol}

Planning for home therapy training includes assessment of the patient and his or her partner's ability to cope with self administration of an intravenous infusion at home. For home therapy to be considered there must be agreeand there must have been no adverse reactions to immunoglobulin in the preceding six months. ${ }^{11}$ The immunology nurse attends the patient's home to supervise the first infusion only. Arrangements are made for delivery of the immunoglobulin product, giving sets and needles etc to the home on a regular basis.

\section{Side effects of immunoglobulin infusions} Symptoms pertaining to any IVIg administration are graded as mild, moderate, and severe. Mild reactions are headache, flushing, lower back pain, myalgia, nausea, chills, and abdominal pain. Moderate reactions include bronchospasm, chest pain, and worsening of mild symptoms. Anaphylaxis constitutes a severe ment with the patient's general practitioner, 
reaction. The safety of self infusion at home has been monitored for over 10 years in the United Kingdom; a recent report has confirmed this to be the case. ${ }^{12}$

The incidence of side effects in patients selected for home therapy is low, but may be related to infusion speed, so correct administration rate is essential. Concurrent infectionsfor example, of the upper respiratory tract-also increase the incidence of side effects, and IVIg therapy should be postponed if the patient is currently infected. In a study of side effects of IVIg home therapy in primary immune deficient patients, ${ }^{12}$ infusion related reactions only occurred in $0.7 \%$ of infusions.

\section{Potential problems associated with high dose IVIg protocols}

The side effects of high dose IVIg have been reviewed extensively. ${ }^{13}$ There are theoretical problems that can arise during a high dose IVIg regimen, although in practice their incidence is low. These include haematological, renal, and neurological problems. Potential haematological problems are haemolysis due to passive transfer of high titre antiblood group antibodies and an increase in plasma viscosity, necessitating caution in infusing elderly patients with incipient cerebovascular disease. Renal complications have included acute renal failure in a patient with mixed essential cryoglobulinaemia, ${ }^{14}$ and a transient rise in serum creatinine.$^{15}$ This may be due to increased tubular load from the sugars present in the immunoglobulin preparation used. ${ }^{16}$ For this reason a rapidly increasing serum creatinine is a contraindication to high dose IVIg therapy. There are case reports ${ }^{17}$ of aseptic meningitis associated with high dose IVIg therapy. The infusion rate in these cases was not stated, and headache may be a feature of IVIg therapy if the recommended infusion rate is exceeded. However, these events are associated with the initial infusions and are not relevant to home treatment unless the patient's clinical condition changes radically, which would negate self infusion anyway.

\section{Monitoring}

The continued use of IVIg is expensive, time consuming, and not without risk. It is therefore essential to monitor progress and to show an objective improvement before deciding to continue with maintenance therapy.

Patients with chronic inflammatory demyelinating polyneuropathies require IVIg at regular intervals, traditionally in the form of a $2 \mathrm{~g} / \mathrm{kg}$ dose given over five days, repeated at variable intervals. There are no solid data at present to guide the dose of IVIg in terms of total IgG serum concentrations, or antibody concentrations to various gangliosides, such as antiGM1. Each patient's response is individual, and the total IgG concentration does not correlate with response, even within the same patient (see figure). A daily functional strength diary, designed on an individual patient basis, has enabled each patient to detect their own early warning signs that the weakness is returning. The patient chooses a number of reproducible daily activities, such as the ability to get out of a bath, to lift a kettle full of water, or to write their name, and grades these on an arbitrary scale, from no function through to full function. Ideally, borderline activities are chosen which were not possible before treatment with IVIg, but which have been regained since starting therapy. This threshold monitoring is more sensitive than gross strength testing. The aim of such monitoring is to prevent reduction in strength before the next scheduled dose of immunoglobulin.

Before each infusion, a sample of blood is taken to measure the preinfusion concentration of IgG (to ensure stable immunoglobulin trough concentrations), and to monitor liver function tests (in view of the potential for hepatotoxic virus infection from the immunoglobulin product).

\section{Maintenance}

There are two variables that can be adjusted to find the optimal immunoglobulin schedule: the dose and the interval between doses. We aim to treat with $1-2 \mathrm{~g} / \mathrm{kg}$ of immunoglobulin, within the half life of immunoglobulin, which is about three to four weeks, but do not give more than $1 \mathrm{~g} / \mathrm{kg}$ in a 24 hour period. Some subjects require uniformly high serum IgG concnentrations, as much as $40-50 \mathrm{~g} / 1$ before an effect is obtained.

Once a stable dose has been established, conversion from hospital therapy to home therapy is done in a graded fashion, with formal teaching in IVIg preparation, safety, adverse reactions, cannulation, and administration. Extensive liaison is established between the patient and their neurologist, immunologist, general practitioner, and specialist immunology nurse.

\section{Conclusions}

Self administration of high dose IVIg at home, prescribed according to a daily functional strength diary kept by the patient, is a feasible and effective technique. It is well liked by patients, and is associated with excellent control of syptoms in chronic motor neuropathies. Side effects are infrequent, and treatment time for the patient in terms of visits to hospital and time actually spent infusing are minimised. Criteria for selection include:

- Established efficacy of IVIg in the patient

- Six month period of IVIg treatment free of adverse reactions

- Suitability of patient to manage self infusion.

Selected patients should only infuse at home after formal training in a recognised centre under the overall supervision of a specialist immunology nurse. ${ }^{18}$

\footnotetext{
1 Ver Meulen M, van Doorn PA, Brand A, Strengers PF, Jennekens FG. Busch, HF. Intravenous immunoglobulin treatment in patients with chronic inflammatory demyelinating polyneuropathy: a double blind, placebo co

2 Dyck PJ, Litchy WJ, Kratz KM, Suarez GA, Low PA, Pineda AA, et al. A plasma exchange versus immune globulin infusion trial in chronic inflammatory demyelinating polyradiculopathy. Ann Neurol 1994; 36: 838-45.
} 
3 van der Meché FGA, Schmitz PIM. Dutch Guillain-Barré Syndrome Group. A randomised trial comparing
intravenous immunoglobulin and plasma exchange in intravenous immunoglobulin and plasma exchange in

4 Bain P, Ellington, C Goodger, E Misbah, S Pangyres, P MacPherson, et al. A randomised double-blind controlled study of intravenous immunoglobulin in the Lambert Eaton syndrome [abstract]. I Neurol Neurosurg Psychiatry 1994;57:1287.

5 Dalakas MC, Illa I, Dambrosia JM, Soueidan SA, Stein DP, Otero C. Dinsmore ST. A controlled trial of high-dose intravenous immunoglobulin infusions as treatment of dermatomyositis. N Engl F Med 1993;329:1993-2000.

6 Donaghy M, Mills KR, Boniface SJ, Simmons J, Wright I, Gregson N. Jacobs J. Pure motor demyelinating polyneuropathy: deterioration after steroid treatment and improvement with intraveous immunoglobulin. I Neurol improvement with intraveous immu
Neurosurg Psychiatry 1994;57:778-83.

7 Azulay J-P, Bun O, Pouget J, Boucraut J, Billé-Turc F, Carles $\mathrm{G}$, Serratrice G. Intravenous immunoglobulin treatment in patients with motor neuron syndromes associated with patients with motor neuron syndromes associated with
anti-GM1 antibodies: a double-blind, placebo controlled anti-GM1 antibodies: a double-blind

8 Chaudhry V, Corse AM, Cornbiath DR, Kuncl RW, Drachman DB, Freimer ML, et al. Multifocal motor neuropathy: response to human immune globulin. Ann Neurol 1993;33: 237-42

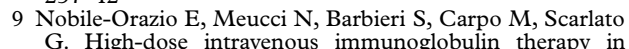
multifocal motor neuropathy. Neurology 1993;43:537-44.
10 van Den Berg LH, Kerkhoff H, Oey PL, Franssen H, Mollee I, Vermeulen $\mathrm{M}$, et al. Treatment of multifocal motor neuropathy with high dose intravenous immunoglobulins: a double blind, placebo controlled study. F Neurol Neurosurg Psychiatry 1995;59:248-52.

11 Chapel HM, Brennan VM. Delson E. Immunoglobulin replacement therapy by self-infusion at home. Clin Exp Immunol 1988;73:160-2.

12 Brennan VM, Cochrane S, Fletcher C, Hendy D, Powell P. Surveillance of adverse reactions in patients self-infusing intravenous immunoglobulin at home. $f$ Clin Immunol 1995;15:116-9.

13 Misbah SA, Chapel HM. Adverse effects of intravenous immunoglobulin. Drug Safety 1993;9:254-62.

14 Barton JC, Herrera GA, Galla JH, Bertoli LF, Work J. Acute cryoglobulinemic renal failure after intravenous infusion of gammaglobulin. Am $\mathcal{F}$ Med 1987;82:624-9.

15 Schifferli J, Leski M, Favre H, Imbach P, Nydegger U. High dose intravenous IgG treatment and renal function. Lancet 1991;337:457-8.

16 Tan E, Hajinazarian M, Bay W, Neff J, Mendell JR. Acute renal failure resulting from intravenous immunoglobulin therapy. Arch Neurology 1993;50:137-9.

17 Rao SP, Teitlebaum J, Miller ST. Intravenous immune globulin and aseptic meningitis. Americam fournal of Diseases of Childhood 1992;146:539-40.

18 Chapel H. Consensus on diagnosis and management of primary antibody deficiencies. BMF 1994;308:581-5. 